Sharif University of Technology
Scientia Iranica
SCIENTIA

\title{
Effect of anisotropy and piezoelectricity on the force-frequency coefficient of AT-cut quartz crystals
}

\author{
M.M. Mohammadi*, H. Daneshpajooh and M. Hamedi \\ School of Mechanical Engineering, College of Engineering, University of Tehran, P.O. Box 11155/4563, Tehran, Iran.
}

Received 8 May 2015; received in revised form 28 September 2015; accepted 3 November 2015

KEYWORDS
Quartz crystal
resonator;
Force-frequency effect;
Anisotropy;
Piezoelectricity.

\section{Introduction}

Quartz crystal resonators (QXRs) have attractive properties for sensor applications. Some of these properties are high resolution, good long-term and short-term stability, minimum hysteresis and creep, and digital output [1-3]. On the other hand, quartz is a material with some temperature compensated cuts which make QXRs widely used as the sensing elements in force and pressure sensors [4-7]. The force detection mechanism depends on the force-frequency effect. This effect is responsible for the change in the resonant frequency of a quartz resonator caused by two diametrically opposed point forces applied on the perimeter of the resonator.

\footnotetext{
*. Corresponding author.

E-mailaddresses: mmmohamamdi@ut.ac.ir (M.M. Mohammadi); hossein.daneshpajooh@gmail.com (H. Daneshpajooh); mhamedi@ut.ac.ir (M. Hamedi)
}

Force-frequency effect is due to the nonlinear elastic properties which couple the initial stress of the applied forces to the resonant vibrations [8].

Rajitsky defined force-frequency coefficient and measured this coefficient for singly rotated quartz crystals as a function of force azimuth angle [9]. Dauwalter described the effect of temperature change on forcefrequency coefficient. He demonstrated that the temperature dependence of force-frequency coefficient is determined by the azimuth angle $\psi$ between the $X$ crystallographic axis and the direction of application of the force [10]. Errnisse measured the force-frequency coefficient of AT-cut for various force azimuth angles at $25^{\circ} \mathrm{C}$ and $78^{\circ} \mathrm{C}$ [11]. His experiments show that the maximum variation of force-frequency coefficient measured at $25^{\circ} \mathrm{C}$ and $78^{\circ} \mathrm{C}$ is about ten percent of maximum value of force-frequency coefficient at these temperatures. More recently, researchers investigated the frequency shifts of degenerate thickness-shear 
modes, and magnetically induced transverse forcefrequency effect in an AT-cut quartz crystal $[12,13]$.

The problem of frequency shift due to external perturbations, like application of external forces or accelerations, belongs to the general theory of incremental elastic deformations superimposed on initial finite deformations [14]. Lee developed the first successful theoretical analysis of the force-frequency effect [15]. Lee derived an explicit formula to predict the frequency changes by employing the theory of incremental elastic deformations superimposed on initial finite deformations. He used Mindlin's general procedure of power-series expansions of displacements and body forces [16]; however, he neglected the piezoelectricity of quartz. Wang modified the Lee equation to include the piezoelectric effect. He successfully solved the forcefrequency problem for incomplete quartz resonator disks. He revealed that the effect of piezoelectricity on force-frequency coefficient of incomplete quartz resonating disk is about 7 percent when the force azimuth angle is zero [17]. In addition to Mindlin procedure, other analytical methods like perturbation method, developed by Tiersten [18], and full numerical methods are used to determine frequency shift in quartz resonators due to external perturbations [14,19-23]. The accuracy of these methods is acceptable, but they are complicated and time consuming. In using the equations proposed by Lee, accurate determination of force-frequency coefficient depends on the order of approximation in power series expansion, accuracy of initial stress and strains, and consideration of piezoelectricity. An analytical solution to the initial stress field for the singly and doubly rotated crystal plates is proposed by Janiaud et al. [24]. We show, in this paper, that this analytical model lacks accuracy in predicting stress values in the resonating disk and does not correlate with the experimental work by Ratajski.

A review of published literature shows that researchers usually neglect the anisotropy and piezoelectricity in analytical calculation of force-frequency coefficient and these effects have not been quantified accurately. It is clear that the effect of anisotropy and piezoelectricity of quartz on the accuracy of force-frequency coefficient merits further investigation. Moreover, in order to predict the force-frequency coefficient at high temperatures, we need models with enough degrees of accuracy that consider effect of temperature change on force-frequency shift.

In this paper, we consider the piezoelectricity and anisotropy effects and adopt Lee formula using Mindlin general procedure to increase accuracy of the results. Also, FEM solution besides Janiaud analytical solution is applied to calculate initial stress and strain distribution for the quartz circular disk. Then, the force-frequency coefficient is calculated as a function of azimuth angle using the formulae proposed by Lee and
Wang [25]. Moreover, a finite element code, based on nonlinear Lagrangian formulation, has been developed to simulate force-frequency effect in AT-cut quartz crystals. The governing equations in FEM model are similar to the equations presented in $[14,25,26]$. By comparing the results of the mathematical models and finite element code with the available experimental data, the effects of anisotropy and piezoelectricity on the force-frequency coefficient are quantified and the validity of Janiaud analytical solution is investigated.

\section{Analytical models for force-frequency effect in quartz}

The change in the resonance frequency of quartz by application of diametric forces is referred to as force-frequency effect. This effect is caused by nonlinear elastic behavior in quartz, which can couple any external perturbation to the elastic vibration. Under this coupling, the wave velocity and the crystal size are changed. Thus, the resonator resonance frequency will change [11]. To quantify force-frequency effect, Ratajski defined force-frequency coefficient for circular piezoelectric disks in 1965 [9]. He carried out some standard tests to measure force-frequency coefficients for singly rotated quartz crystals. Ratajski forcefrequency coefficient, $K_{f}$, is defined as:

$$
\frac{\Delta f}{f_{0}}=K_{f} \frac{F N_{0}}{D t},
$$

where $F$ is applied force, $\Delta f / f_{0}$ is the fractional frequency shift, $N_{0}$ is the frequency constant, $D$ is the plate diameter, and $t$ is its thickness. The frequency constant equals half the acoustic velocity of propagating wave along the thickness of the crystal. For ATcut crystal, the frequency constant for thickness shear wave is $1664 \mathrm{kHz} . \mathrm{mm}$. Figure 1 shows the circular disk which is subjected to diametric opposed forces. Azimuth angle, $\psi$, defines the location where the force is applied on the perimeter of disk and variation of $\psi$ changes the force-frequency coefficient.

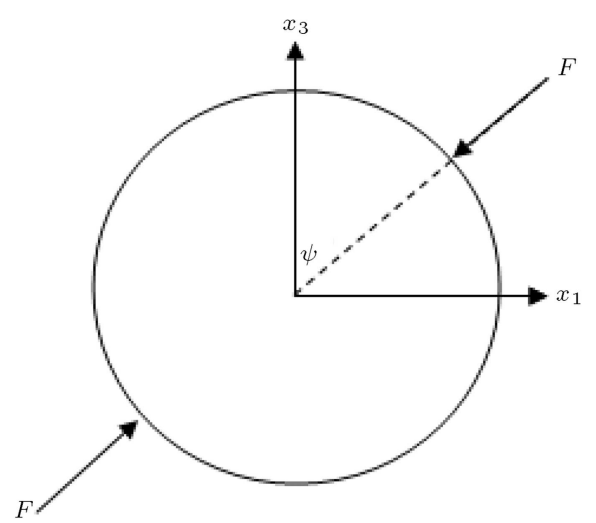

Figure 1. Circular quartz disk subjected to two opposed diametric forces. 
One of the main methods to analyze the forcefrequency effect has been proposed by Lee [15]. Lee derived the basic equations of the theory in Lagrangian formulation to analyze the force-frequency effect at room temperature. In the nonlinear stress-strain relations, third-order elastic coefficients were included. He derived equations of motions from the variational principle of elasticity. Mindlin general procedure of power series expansion, in variational principle for the three-dimensional elasticity, was utilized to obtain the eigen-frequencies. Lee developed an explicit formula for predicting the frequency changes of rotated Y-cut quartz crystal subjected to initial mechanical biases as:

$$
\begin{aligned}
\frac{\Delta f}{f_{0}}= & U_{1,1}^{(0)}+\frac{1}{2 C_{66}}\left[C_{661} E_{1}^{(0)}+C_{662} E_{2}^{(0)}\right. \\
& \left.+C_{663} E_{3}^{(0)}+C_{664} E_{4}^{(0)}\right]
\end{aligned}
$$

where $\Delta f / f_{0}$ is the variation of fundamental thickness shear frequency, and $f_{0}$ is fundamental cut-off frequency of $Y$-cut quartz, $U_{1,1}^{(0)}$ is zero-order component of initial displacement, $E_{r}^{(0)}$ is zero-order initial strain, and $C_{66}$ and $C_{66 r}$ are the second- and third-order elastic constants of AT-cut quartz crystal, respectively.

Lee neglected the piezoelectric effect in incremental stress-strain and potential energy density relations. Wang modified Lee's theory to include the piezoelectric effect [17]. He derived an explicit formula for forcefrequency coefficient of AT-cut crystal. Lee neglected the piezoelectric effect in incremental stress-strain and potential energy density relations. Wang modified Lee's theory to include the piezoelectric effect [17]. He derived an explicit formula for force-frequency coefficient of AT-cut crystal. This formula is:

$$
\begin{aligned}
\frac{\Delta f}{f_{0}}= & U_{1,1}^{(0)}+\frac{1}{2 C_{66}}\left[C_{661} E_{1}^{(0)}+C_{662} E_{2}^{(0)}\right. \\
& \left.+C_{663} E_{3}^{(0)}+C_{664} E_{4}^{(0)}\right] \\
& +\frac{e_{62}\left(e_{62}+e_{21}\right)\left(1+2 U_{1,1}^{(0)}\right)}{2 \kappa^{2} C_{66}\left(\varepsilon_{22}+\varepsilon_{23}\right)}
\end{aligned}
$$

where $e_{i j}$ and $\varepsilon_{22}$ are piezoelectric and dielectric coefficients, respectively, and $\kappa$ is the correction factor which equals $\pi / \sqrt{12}$. $\kappa$ compensates the approximation error in calculation of the frequency of thickness shear mode of vibration. This error arises from the approximation included in power series expansion of thickness shear deformation along the thickness of plate. More details on the calculation of $\kappa$ can be found in [15].

\section{Governing equations for initial stress and strain}

The anisotropic structure of quartz in determination of stress is not usually considered in the published work. Considering an isotropic circular disk subjected to two opposed diametrical forces, stress components at the center of the disk are:

$$
\begin{aligned}
& T_{11}=\frac{-2}{\pi} \frac{F}{t D}(1+2 \cos 2 \psi)=T_{x x}, \\
& T_{33}=\frac{-2}{\pi} \frac{F}{t D}(1+2 \cos 2 \psi)=T_{z z}, \\
& T_{13}=\frac{-4}{\pi} \frac{F}{t D} \sin 2 \psi,
\end{aligned}
$$

and the strain components can be calculated by:

$$
\begin{aligned}
& E_{1}=S_{11} T_{11}+S_{13} T_{33}+S_{15} T_{13}, \\
& E_{2}=S_{21} T_{11}+S_{23} T_{33}+S_{25} T_{13}, \\
& E_{3}=S_{31} T_{11}+S_{33} T_{33}+S_{35} T_{13}, \\
& E_{4}=S_{41} T_{11}+S_{43} T_{33}+S_{45} T_{13} .
\end{aligned}
$$

Then, zero-order strain can be obtained by [15]:

$$
T_{i}^{(0)}=t T_{i}=t C_{i j} E_{j}^{(0)} .
$$

In the above equations, $S_{i j}$ and $T_{i j}$ are compliance and stress components, respectively. Using Eqs. (4)-(6), the components of zero-order strains can be obtained at desired azimuth angle.

\subsection{Calculation of stress with anisotropic assumption}

Due to anisotropic characteristics of quartz, the stress distribution, induced by diametrical forces in quartz resonators, is not homogeneous and is difficult to determine. Janiaud developed an analytical method to calculate the second-order approximate stress distribution in anisotropic circular disk induced by a concentrated force [24]. Upon this model, stress components for rotated $Y$-cut at the center of the disk are:

$$
\begin{aligned}
& T_{1}=-\frac{F}{\pi}\left(\kappa_{1}^{(0)}=\frac{1}{8}\left(\frac{\lambda R^{3}}{C}+\frac{\lambda R^{3}}{D}\right)\right), \\
& T_{3}=-\frac{F}{\pi}\left(\kappa_{3}^{(0)}\right) \\
& T_{5}=0
\end{aligned}
$$

where $k_{1}^{(0)}, k_{3}^{(0)}, \lambda, C$, and $D$ are function of material constants, which can be found in [24], and $R$ is the radius of the disk. 


\section{Finite element modeling of force-frequency effect}

Along with using the mathematical model, a finite element code is developed to investigate the forcefrequency effect in AT-cut quartz using the multiphysics software, COMSOL 4.3. This software provides a finite element package which gives users the required flexibility to write their own partial differential equations and to modify the equation of motions.

Lee and Yong [25] have shown that in small deformation, the nonlinear governing equations of quartz can be simplified by modeling it in three steps. According to this assumption, the model includes two main sub-models, the initial model, and the incremental model. The initial model relates the natural states of material to an intermediate state, using Lagrangian formulation. Using the nonlinear governing equation of an anisotropic material (Eqs. (8)-(11)), the initial model investigates the external loading. The initial three-dimensional nonlinear governing equations for quartz, adopted in FEM model, are [25]:

$$
\begin{aligned}
& E_{i j}=\frac{1}{2}\left(U_{j, i}+U_{i, j}+U_{k, i} U_{k, j}\right), \\
& T_{i j}=C_{i j k l}^{\theta} E_{k l}+\frac{1}{2} C_{i j k l m n}^{\theta} E_{k l} E_{m n}, \\
& \rho_{0} \ddot{U}_{i}=\left(T_{i j}+T_{j k} U_{i, k}\right), j \quad \text { in } \quad V, \\
& P_{i}=n_{j}\left(T_{i j}+T_{j k} U_{i, k}\right) \quad \text { in } \quad S
\end{aligned}
$$

where $E_{i j}$ is the strain, $T_{i j}$ is the stress, $U_{i, j}$ is the displacement gradient, $P_{i}$ is the surface traction in the boundary surfaces, and $C_{i j k l}^{\theta}$ and $C_{i j k l m n}^{\theta}$ are secondand third-order stiffness coefficients, respectively.

The initial stress, initial strain, and initial displacement, which can be derived by stationary study of quartz in the initial model, are considered as the incremental model inputs. Therefore, the incremental model uses linear equations (Eqs. (12)-(15)) to obtain the resonant frequencies by harmonic responses [26,27]. The governing equations for the incremental model are as follows:

$$
\begin{aligned}
& e_{i j}=\frac{1}{2}\left(u_{j, i}+u_{i, j}+u_{k, i} U_{k, j}+U_{k, i} u_{k, j}\right), \\
& t_{i j}=C_{i j k l}^{\theta} e_{k l}+\frac{1}{2} C_{i j k l m n}^{\theta} e_{k l} E_{m n}, \\
& \rho_{0} \ddot{u}_{i}=\left(t_{i j}+t_{j k} U_{i, k}+T_{j k} u_{i, k}\right)_{, j} \text { in } V, \\
& p_{i}=n_{j}\left(t_{i j}+t_{j k} U_{i, k}+T_{j k} u_{i, k}\right) \text { on } S .
\end{aligned}
$$

The piezoelectricity effect can be implemented by coupling these equations with electrical displacement.
Using the overall stress-strain piezoelectric model along with Gauss law, Eq. (16), a near-complete model with both piezoelectricity and anisotropic characteristics of quartz is developed:

$$
D_{i, i}=0 \quad \text { in } \quad V
$$

where $D$ is the electrical displacement.

Since the quartz crystals have anisotropic characteristics, and their governing equations are non-linear, they cannot be implemented using the default feature of the FEA software. Subsequently, the whole governing equations and the boundary conditions are converted to weak-form expressions. Thus, the anisotropic characteristics of quartz can be implemented using the PDE (Partial Differential Equation) interference of the software. Also, the electrical charge of the electrodes could be coupled with the structural model to implement the piezoelectricity aspect of quartz crystals. Therefore, the FEM model is expected to produce more accurate results.

The thickness shear mode in quartz crystals is a function of the thickness of the quartz (Figure 2), though the width and length of plate also have effect on thickness shear mode frequency. Therefore, in the investigation of force-frequency effect, a swept mesh is utilized to control the mesh number in thickness direction. Our final model includes 2094 Lagrangian quadratic elements.

The resonance behavior of quartz is considered to be harmonic. Therefore, the governing equations will take the form of:

$$
u_{i}=\bar{u}_{i} e^{-i \omega t}
$$

AT-cut, thickness shear mode, displacement along $x$

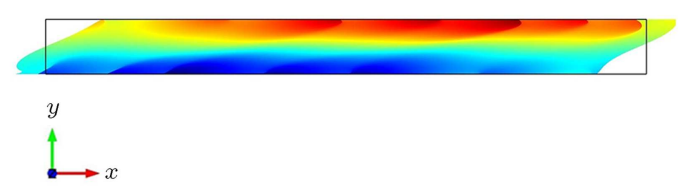

AT-cut, thickness shear mode, total displacement $x$

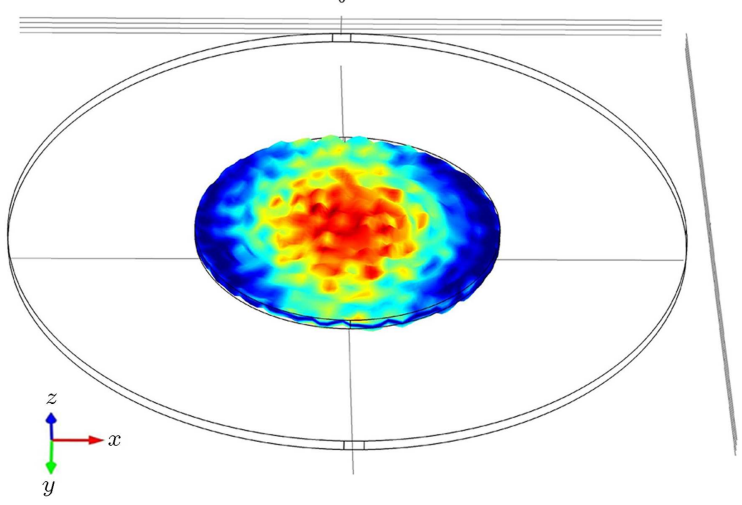

Figure 2. Thickness-shear mode in the AT-cut quartz crystal plate. 


$$
\rho_{0} \omega^{2} \bar{u}_{i}=\left(t_{i j}+t_{j k} U_{i, k}+T_{j k} u_{i, k}\right)_{, j} \quad \text { in } \quad V
$$

where, $\omega$ is the resonance frequency.

\section{Results and discussion}

To calculate force-frequency constants, we considered a circular AT-cut quartz disk with diameter of $10 \mathrm{~mm}$, thickness of $0.1660 \mathrm{~mm}$, and fundamental first thickness shear frequency of $10 \mathrm{MH}$, which was subjected to two diametrically opposed forces. Considering the stress component, $T_{x x}$, to be the stress along $x_{1}$ axis $(x)$ at the center of the disk in Figure 3 , and $T_{z z}$ to be the stress along $x_{3}$ axis $(z)$, we calculated these stress components for every 5 degrees of azimuth angle based on isotropic and anisotropic assumptions. For anisotropic assumption, Janiaud method and finite element solution were used.
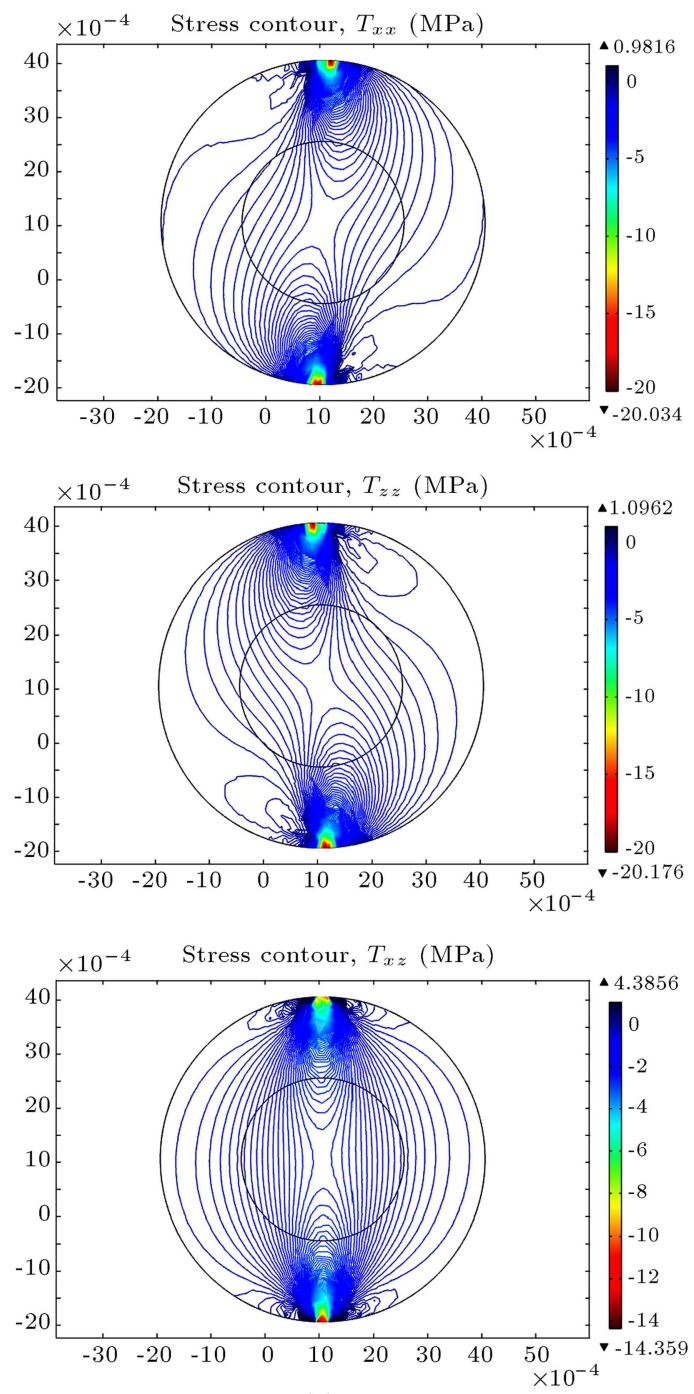

(a)
In order to calculate the initial stresses, based on anisotropic assumption with Janiaud method, the values of $k_{1}^{(0)}, k_{3}^{(0)}, \lambda, C$, and, $D$ were determined by applying the published values of compliance coefficients for AT-cut quartz crystal [26]. Calculated constants were substituted into Eq. (7) to calculate stress components at the center of the disk as a function of azimuth angle. The obtained results for stress components are shown in Figures 4 and 5.

It can be seen in Figure 4 that the sign of stress component, $T_{z z}$, of Janiaud method is opposite to those of other methods. Thus, we considered the modified stress component, $T_{z z}$, in Janiaud model to have a similar sign with the stress component, $T_{z z}$, of isotropic and finite element solutions. The new stress components are labeled as modified Janiaud method in Figure 5.

By substituting the calculated stress components
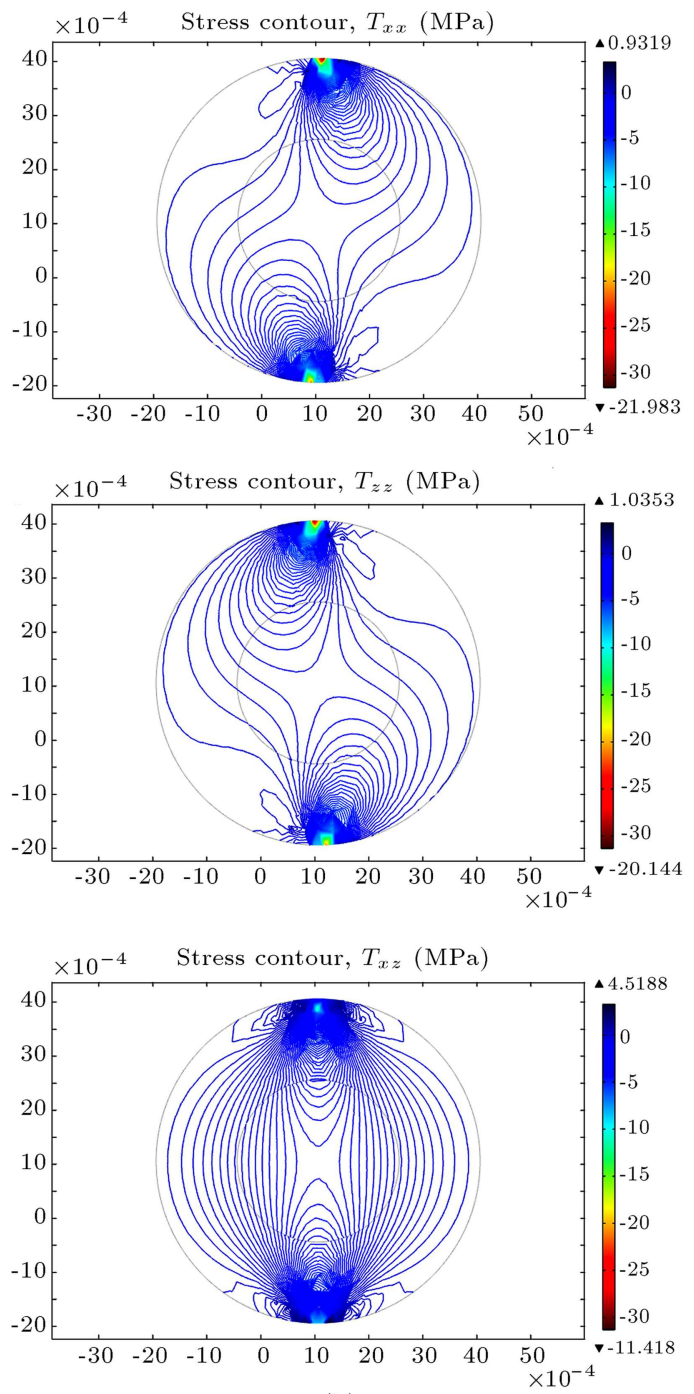

(b)

Figure 3. Stress component distribution in (a) anisotropic, and (b) isotropic models under $1 \mathrm{~N}$ loading at $45^{\circ}$ azimuth angel. 


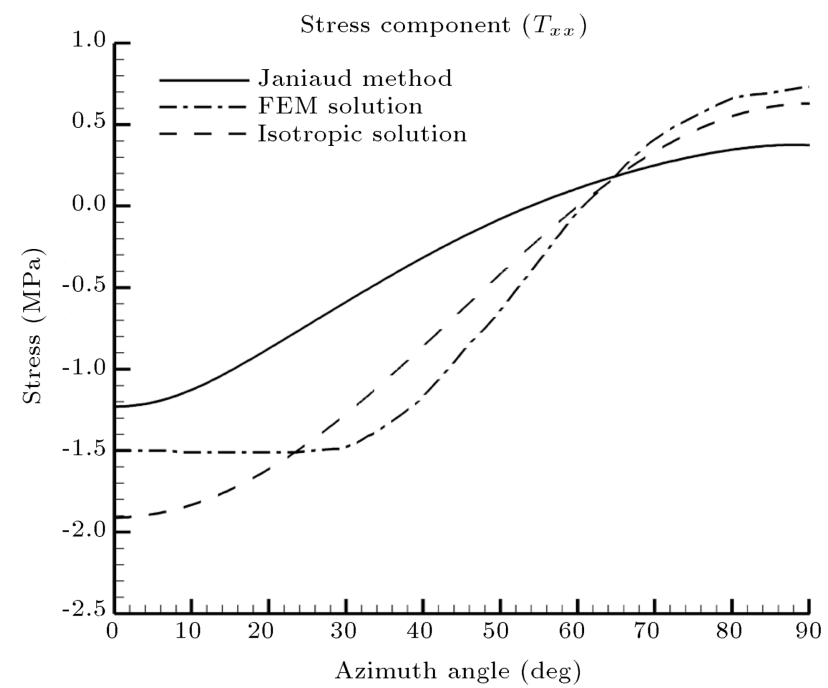

Figure 4. Stress component, $T_{x x}$, as a function of azimuth angle.

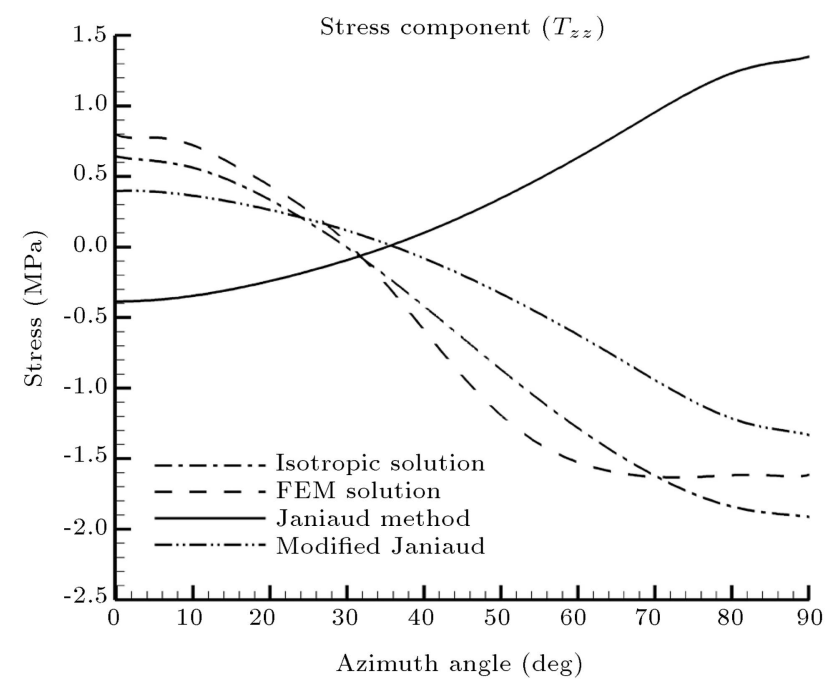

Figure 5. Stress component, $T_{z z}$, as a function of azimuth angle.

from FEM into Eqs. (5) and (6) and then into Eqs. (2) and (3), the values of force-frequency coefficient for both Lee and Wang methods are obtained, which are plotted as a function of force azimuth angle in Figure 6 (labeled as FEM-Lee and FEM-Wang). As can be seen in Figure 6, the results of Lee and Wang models are in good consistency.

Moreover, the calculated stress components from the initial FEM model are implemented in FEM incremental model (FEM stress, $T_{i j}$, strain, $E_{i j}$, and displacement, $\left.U_{i j}\right)$. The incremental model uses the eigenfrequency solver to obtain the resonant frequencies. The predicted values of force-frequency coefficients by FEM method are plotted in Figure 7 as a function of force azimuth angle. Also, to validate the results of force-frequency models, the published experimental values of force-frequency coefficient in Ratjiski [9] for

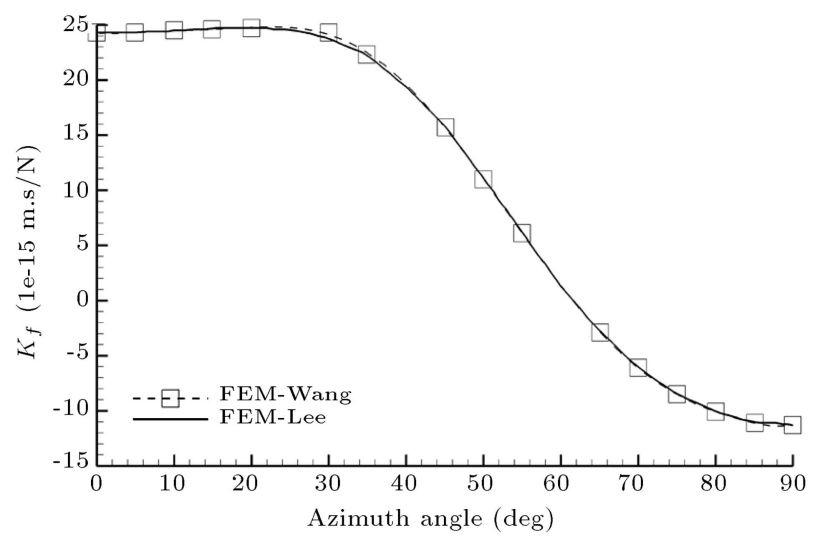

Figure 6. Effect of piezoelectricity on force-frequency coefficient of AT-cut quartz crystal.

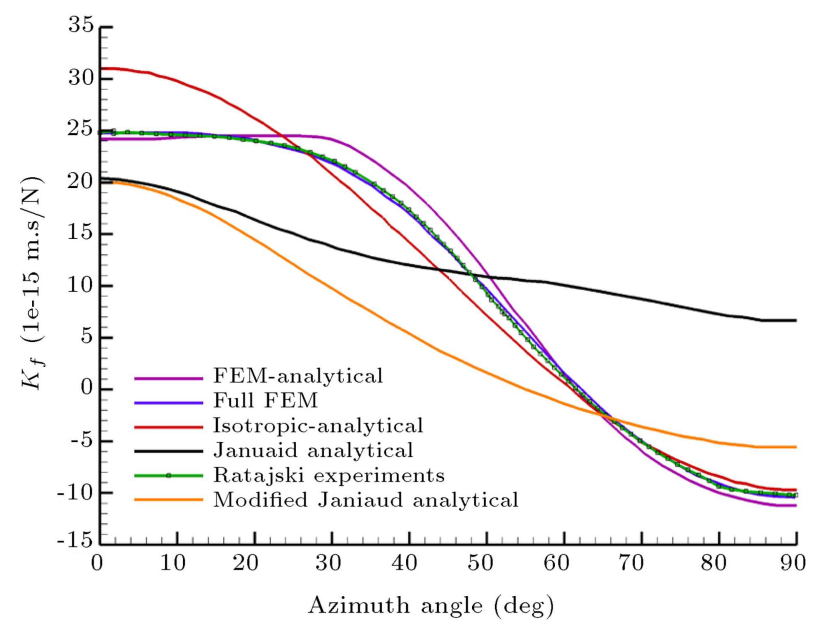

Figure 7. Force-frequency coefficient of AT-cut quartz crystal as a function of azimuth angle.

AT-cut crystal are plotted in Figure 7. As can be seen, the FEM model results are in a good consistency with Ratjiski experimental data. However, the modified Janiaud analytical model resulted in better prediction of force-frequency behavior of quartz than the unmodified model; however, still both models considerably failed to predict it well.

To verify the accuracy of each model quantitatively, the standard error of each model with respect to the experimental values of Ratajski is calculated by the following equation and the results are represented in Table 1:

$$
\text { error }=\left(\frac{1}{n} \sum_{i=1}^{n}\left(y_{i}-y_{e x p}\right)^{2}\right)^{0.5} \text {. }
$$

In Table 1, the first column assigns a number to each force-frequency calculation method; and the second, third, and fourth columns describe the initial stress calculation method, the eigenvalue solution method, and the evaluate least square error, respectively. Methods number one and two relate to Janiaud method 
Table 1. Standard error (m.s/N e-15) of different models used to evaluate force-frequency coefficients.

\begin{tabular}{cllc}
\hline Method & \multicolumn{1}{c}{$\begin{array}{c}\text { Initial stress } \\
\text { solving method }\end{array}$} & \multicolumn{1}{c}{$\begin{array}{c}\text { Eigen value } \\
\text { solver }\end{array}$} & $\begin{array}{c}\text { Total least } \\
\text { square error } \\
\text { (m.s/N E-15) }\end{array}$ \\
\hline 1 & Janiaud model & Lee equation & 43.91 \\
2 & Modified Janiaud model & Lee equation & 32.5 \\
3 & Isotropic mathematical model & Lee equation & 12.4 \\
4 & Isotropic mathematical model & Wang equation & 12.34 \\
5 & Finite element anisotropic model & Lee equation & 5.75 \\
6 & Finite element anisotropic model & Wang equation & 5.66 \\
7 & Finite element anisotropic model & Finite element method & 1.45 \\
\hline
\end{tabular}

of solving for stress components. As can be seen, the modification of the sign stress component, $T_{z z}$, in modified Janiaud method has increased the accuracy. However, this stress model leads to inaccurate forcefrequency coefficients compared with other models.

By considering the anisotropy factor, the standard error using Lee method is decreased from $12.4 \mathrm{E}$ $15(\mathrm{~ms} / \mathrm{N})$ to $5.75 \mathrm{E}-15(\mathrm{~ms} / \mathrm{N})$. As mentioned in the previous section, Wang model was a modification of Lee model to include piezoelectric effect. Comparing the regression errors for these two models shows that the weak piezoelectricity in quartz has a small effect on force-frequency coefficient and can be neglected in most cases. On the other hand, it can be seen that the stresses which are obtained by applying Janiaud procedure lead to lower degrees of accuracy compared to other methods. The relatively high standard error of modified Janiaud model shows that this modification cannot compensate the error of Janiaud model and this analytical model is not accurate enough to predict the stress at the center of the disk.

\section{Conclusions}

The force-frequency coefficient of AT-cut quartz as a function of azimuth angle is predicted using Lee and Wang mathematical models and FEM simulations. The model is validated by comparing the results with published experimental works. In calculations, crystal anisotropy and piezoelectricity were taken into account and the following observations can be made:

1. Taking the anisotropy into account in determining the initial stress bias can enhance the accuracy of force-frequency model and reduce the standard error in predicting force-frequency coefficient from 12.4 E-15 (ms/N) to 5.75 E-15 $(\mathrm{ms} / \mathrm{N})$;

2. The effect of piezoelectric coefficients on values of force-frequency constants is not considerable for AT-cut quartz crystal in most applications;

3. Janiaud analytical model lacks accuracy in predicting stress values in the resonating disk and needs to be revised;
4. The best results can be achieved by full finite element solution and the regression error can be reduced up to $1.45 \mathrm{E}-15(\mathrm{~ms} / \mathrm{N})$ by this method;

5. The theoretical model of force-frequency effect, which has been proposed by Lee, is a suitable model that is able to predict the values of force-frequency coefficients with a high degree of accuracy. This work showed that taking the anisotropic properties of crystal into account is vital to evaluate the initial stresses and strains.

\section{References}

1. Goujon, N., Hori, H., Liang, K.K. and Sinha, B.K. "Applications of piezoelectric materials in oilfield services", IEEE Trans. Ultrason., Ferroelectr. Freq. Control, 59(9), pp. 2042-2050 (2012).

2. Lan, B.Y., Tian, W.J., Zhao, Q.J. and Lv, Y.S. "Study on the design and the stability of integrated quartz resonator", Adv. Mater. Res., 846, pp. 569-573 (2014).

3. Cerda, R.M., Understanding Quartz Crystals and Oscillators, Artech House (2014).

4. Lin, L.D., Qiu, C.J., Yu, X. and Zhou, P. "Equal radial force structure pressure sensor data analysis and finite element analysis", In Intelligent Computation in Big Data Era, pp. 309-319. Springer Berlin Heidelberg (2015).

5. Murozaki, Y. and Arai, F. "Wide range load sensor using quartz crystal resonator for detection of biological signals", IEEE Sens. J., 15(3), pp. 1913-1919 (2015).

6. Murozaki, Y., Nogawa, K. and Arai, F. "Miniaturized load sensor using quartz crystal resonator constructed through microfabrication and bonding", ROBOMECH $J .$, 1(1), pp. 1-7 (2014).

7. Cheng, R., Zhao, Y., Li, C., Tian, B., Yu, Z. and Liu, K. "Design and fabrication of a resonant pressure sensor by combination of DETF quartz resonator and silicon diaphragm", Microsyst. Technol., 21(3), pp. 631-640 (2015).

8. Zhang, H., Turner, J.A., Yang, J. and Kosinski, J.A. "Force-frequency effect of thickness mode langasite resonators", Ultrason., 50(4), pp. 479-90 (2010).

9. Ratajski, J.M. "Force-frequency coefficient of singly 
rotated vibrating quartz crystals", IBM. J., Res., Dev., 12(1), pp. 92-99 (1968).

10. Dauwalter, C.R. "The temperature dependence of the force sensitivity of AT-cut quartz crystals", Proc. 26th. Annu. Freq. Contr. Symp, pp. 108-112 (1972).

11. EerNisse, E.P. "Temperature dependence of the force frequency effect for the AT, FC, SC and rotated XCuts", In Proc. 34th Annu. Freq. Contr. Symp, pp. 426-430 (1980).

12. Bao, Y., Zhang, H. and Kosinski, J. "Experimental measurement of the frequency shifts of degenerate thickness-shear modes in a rotated Y-cut quartz resonator subject to diametrical forces", IEEE Trans. Ultrason., Ferroelectr., Freq. Control, 62(3), pp. 560$564(2015)$.

13. Hatipoglu, G. and Tadigadapa, S. "Experimental studies in magnetically induced transverse force-frequency effect in thin quartz microresonators", J. Appl. Phys., 118(3), pp. 034508 (2015).

14. Chen, J., Yong, Y.K., Kubena, R., Kirby, D. and Chang, D. "On the acceleration sensitivity and its active reduction by edge electrodes in At-cut Quartz resonators", IEEE Trans. Ultrason. Ferroelectr. Freq. Control, 62(6), pp. 1104-1113 (2015).

15. Lee, P.C.Y., Wang, Y.S. and Markenscoff, X. "Highfrequency vibrations of crystal plates under initial stresses", J. Acoust. Soc. Am., 57(1), pp. 95-105 (1975).

16. Mindlin, R.D. "High frequency vibration of piezoelectric crystal plates", Int. J. Solids Structures, 8(7), pp. 895-906 (1972).

17. Wang, Z., Zhu, H., Dong, Y., Wang, J. and Feng, G. "Force-frequency coefficient of symmetrical incomplete circular quartz crystal resonator", IEEE Trans. Ultrason., Ferroelectr. Freq. Control, 48(5), pp. 1471-1479 (2001).

18. Tiersten, H.F. "Perturbation theory for linear electroelastic equations for small fields superposed on a bias", J. of Acoustic Soc. of Amer., 64(3), pp. 832-837 (1978).

19. Wu, R., Wang, J., Du, J., Huang, D., Yan, W. and $\mathrm{Hu}, \mathrm{Y}$. "An analysis of nonlinear vibrations of coupled thickness-shear and flexural modes of quartz crystal plates with the homotopy analysis method", IEEE Trans. on Ultrasonics, Ferroelec. Freq. Contr., 59(1), pp. 30-39 (2012).

20. Yong, Y.K., Patel, M.S., Srivastava, S., Tanaka, M. and Imai, T. "The impact of finite element analysis on the design of quartz resonators", Int. Freq. Contr. Symp. and Exp., Miami, FL (2006).

21. Liu, N., Yang, J., Hu, Y., Chen, X. and Jiang, W., "Frequency shifts in plate crystal resonators induced by electric, magnetic, or mechanical fields in surface films", IEEE Trans. on Ultrasonics, Ferroelec. Freq. Contr., 58(12), pp. 2588-2595 (2011).

22. Shi, J., Fan, C., Zhao, M. and Yang, J. "Variational formulation of the Stevens-Tiersten equation and application in the analysis of rectangular trapped-energy quartz resonators", J. of Acoustical Soc. of Amer., 135(1), pp. 175-181 (2014).

23. Fan, Y., Ji, X., Liu, X. and Cai, P. "The nonlinear analysis of elastic wave of piezoelectric crystal plate with perturbation method", Wave Motion, 51(5), pp. 798-803 (2014).

24. Janiaud, D., Nissim, L. and Gagnepain, J.J. "Analytical calculation of initial stress effect on anisotropic crystal: Application to quartz resonators", In Proc. 32nd Annu. Freq. Contr. Symp, pp. 169-179 (1978).

25. Lee, P.C.Y. and Yong, Y.K. "Temperature derivatives of elastic stiffness derived from the frequencytemperature behavior of quartz plates", J. of App. Phys., 56(5), pp. 1514-1521 (1984).

26. Patel, M.S., Nonlinear Behavior in Quartz Resonators and Its Stability, Ph.D. Dissertation, Dept. Civil. Eng., New Brunswick., New Jersey Univ. (2008).

27. Beerwinkle, A.D. "Nonlinear finite element modeling of quartz crystal resonators", M.S. Thesis, Dept. Mech. Eng., Oklahoma State Univ, Stillwater, USA (2011).

\section{Biographies}

Mohammad Mostafa Mohammadi is currently a PhD student in Mechanical Engineering School, University of Tehran. He obtained his MS degree in Manufacturing Engineering from Tarbiat Modares University of Tehran, in 2007, and BS degree from Tabriz University, in 2004. His $\mathrm{PhD}$ thesis is on mathematical modelling of quartz resonator pressure sensors.

Hossein Daneshpajooh received the BS degree in Mechanical Engineering from Amirkabir University of Technology, in 2008. He is now an MS student in Mechanical Engineering School, University of Tehran. His current research is focused on analytical investigation of micro/nano systems and smart material such as piezoelectric devices.

Mohsen Hamedi is Professor of Manufacturing at University of Tehran, where he teaches various courses in graduate and undergraduate levels. His research interests are microfabrication, microsystems, and optimization of manufacturing processes. He has published more than 130 papers in international journals and conferences along with supervising more than 50 doctorate and master theses. He obtained his BSc and MSc from University of Tehran and PhD from University of New Brunswick, Canada. He is a founding member of Iranian Society of Value Engineering, The R\&D Society of Iranian Industry, Iranian Society of Manufacturing Engineers, and a member of Iranian Society of Mechanical Engineers. 